\title{
End-to-end Design of a PUF-based Privacy Preserving Authentication Protocol ${ }^{\star}$
}

\author{
Aydin Aysu ${ }^{1}$, Ege Gulcan ${ }^{1}$, Daisuke Moriyama ${ }^{2}$, Patrick Schaumont ${ }^{1}$, and \\ Moti Yung ${ }^{3}$ \\ 1 Virginia Tech, USA \\ \{aydinay, egulcan, schaum\}@vt.edu \\ 2 NICT, Japan \\ dmoriyam@nict.go.jp \\ 3 Google Inc. and Columbia University, USA \\ motiyung@gmail.com
}

\begin{abstract}
We demonstrate a prototype implementation of a provably secure protocol that supports privacy-preserving mutual authentication between a server and a constrained device. Our proposed protocol is based on a physically unclonable function (PUF) and it is optimized for resource-constrained platforms. The reported results include a full protocol analysis, the design of its building blocks, their integration into a constrained device, and finally its performance evaluation. We show how to obtain efficient implementations for each of the building blocks of the protocol, including a fuzzy extractor with a novel helper-data construction technique, a truly random number generator (TRNG), and a pseudo-random function (PRF). The prototype is implemented on a SASEBO-GII board, using the on-board SRAM as the source of entropy for the PUF and the TRNG. We present three different implementations. The first two execute on a MSP430 soft-core processor and have a security level of 64-bit and 128-bit respectively. The third uses a hardware accelerator and has 128-bit security level. To our best knowledge, this work is the first effort to describe the end-to-end design and evaluation of a privacy-preserving PUF-based authentication protocol.
\end{abstract}

Keywords: Physically Unclonable Function, authentication, privacy-preserving protocol, implementation

\section{Introduction}

Physically Unclonable Functions (PUFs) have been touted as an emerging technology to support authentication of a physical platform. However, the design of PUF-based authentication protocols is complicated, and many pitfalls have been identified with existing protocols [10]. First, many protocols are ad-hoc designs. In the absence of a formal adversary model, one can only hope that no security

\footnotetext{
* The preliminary version of this paper was presented in CHES 2015 [2].
} 
holes are left. Second, while theoretical security models may provide assurance on the achieved level of security, these models typically lack a consideration of implementation issues. The cryptographic engineering of a PUF-based authentication protocol requires more than a formal proof. Finally, typical PUF-based protocol designs assume ideal PUF behaviors. They make abstraction of complex noise effects that come with real PUF. The actual performance of these protocol designs, and often also their implementation cost, remains unknown.

We believe that these issues can be systematically addressed, by combining a theoretical basis with sound cryptographic engineering [6]. In this paper, we aim to demonstrate this for a PUF-based privacy-friendly authentication protocol.

There are many PUF-based protocols that claim privacy [7, 25, 37, 21, 23]. We observed that most of these earlier proposals do not have a formal proof of security and privacy. In our opinion, a formal basis is required to clarify the assumptions of the protocol. For example, a recent analysis by Delvaux et al. [10] showed that only one [37] of these privacy-claiming PUF protocols actually provides privacy. Furthermore, none of the earlier proposed PUF-based protocols disclosed an implementation and a performance evaluation. This is required, as well, because the security and privacy properties of a PUF-based protocol are directly derived from the PUF design. These two reasons are the direct motivation for our protocol design, and its evaluation.

A PUF, a central element of our design, returns noisy data and uses a fuzzy extractor (FE) to ensure a reliable operation. The fuzzy extractor associates helper data with every PUF output to enable reconstruction of later noisy PUF outputs. However, the generation of helper data (Gen) and the reconstruction of a PUF output (Rec) are algorithms with asymmetric complexity: helper data generation has lower complexity than PUF output reconstruction. Realizing this property, van Herrewege et al. proposed reverse fuzzy extractors, which place the helper data generation within the constrained device [38]. However, the original reverse fuzzy-extractor protocol does not offer privacy. To achieve this objective, we rely on a protocol design by Moriyama et al. [30]. Assuming that a PUF is tamper-proof, their design leaves no traceable information within the device. This is achieved by using a different PUF output at every authentication, and thus by changing the device credential after every authentication.

Our proposed protocol starts from this design, and adapts it for a reverse fuzzy-extractor implementation. We maintain the formal basis of the protocol, but we also provide a detailed implementation and evaluation.

We note that there are contextual elements to privacy that are not addressed by our protocol. For example, we cannot offer privacy against an adversary who can physically trace every device in between authentications, or who can use other (non-cryptographic) mechanisms to identify a device [26]. These are context-dependent elements which have to be addressed by the application.

Compared to earlier work, we claim the following innovative features:

Novel Protocol. Our protocol merges privacy with a reverse fuzzy-extraction design, and is therefore suited for implementation on constrained platforms that also need privacy. Our protocol supports mutual (device-first) authentication. 
End-to-end Design. We demonstrate a complete design trajectory, from provably secure protocol specification towards performance evaluation. We are not aware of any comparable efforts for other protocols. While other authors have suggested possible designs $[29,27,38]$, the actual implementation of such a protocol has, to our knowledge, not yet been demonstrated.

Interleaved Error Correction. We present a novel technique for efficient helper data generation using an interleaved $\mathrm{BCH}$ code, as well as its security analysis. Our decoding strategy is computationally simple, and enables the use of a single $\mathrm{BCH}(63,16,23)$ primitive while still achieving $10^{-6}$ overall error rate.

The end-to-end design of a PUF-based protocol covers protocol design, protocol component instantiation, architecture design, and finally evaluation of cost and performance. We build our prototype on top of a SASEBO-GII board, using the resources available on the board to construct the PUF and the protocol engine. We use the 2Mbit SRAM on the SASEBO-GII board as the source of entropy. We construct the following protocol components: an SRAM PUF, an SRAM TRNG, a pseudorandom function (PRF) design using the SIMON block cipher, and a fuzzy extractor based on an interleaved $\mathrm{BCH}$ error corrector and a PRF based strong extractor. We provide a design specification at two security levels, 64-bit and 128-bit.

Next, we implement these protocol components using an MSP430 processor (mapped as a soft-core on the SASEBO-GII board), an SRAM and a non-volatile memory. We also design a hardware accelerator to handle all cryptographic steps of the protocol, including the PRF, message encryption, and PUF output coding. Then, we implement the server-functionality on a PC connected to the SASEBOGII board, and characterize the performance of the implementation under an actual protocol execution.

The remainder of this paper is organized as follows. Section 2 introduces the privacy preserving authentication protocol, describing its security assumption and important features. Section 3 describes the design of the protocol components: the SRAM PUF, the SRAM TRNG, the PRF, and the fuzzy extractor. Section 4 discusses the prototype implementation of the protocol, covering the system-level (server and device), the device platform, and the accelerator hardware engine. Section 5 presents the results, including implementation complexity and cost. We conclude the paper in Section 6 .

\section{Secure and Private PUF-based Authentication Protocol}

In this section, we describe the protocol notation, the assumed trust model, and the flow of the overall PUF protocol. We describe its main features in this section and security analysis of this protocol including security proof is found in Appendix A.

\subsection{Notation}

When $A$ is a set, $y \stackrel{\cup}{\leftarrow} A$ means that $y$ is uniformly selected from $A$. When $A$ is a deterministic algorithm, $y:=A(x)$ denotes that an output from $A(x)$ with input 
$x$ is assigned to $y$. When $A$ is a probabilistic machine or an algorithm, $y \stackrel{R}{\leftarrow} A(x)$ denotes that $y$ is randomly selected from $A$ according to its distribution. $\operatorname{HD}(x, y)$ denotes the Hamming distance between $x$ and $y . \bar{H}_{\infty}(x)$ denotes the min-entropy of $x$. In addition, we use the following notations for cryptographic functions throughout the paper.

(Truly Random Number Generator) TRNG derives a truly random number sequence.

(Physically Unclonable Functions) $f: \mathcal{K} \times \mathcal{D} \rightarrow \mathcal{R}$ which takes as input a physical characteristic $x \in \mathcal{K}$ and message $y \in \mathcal{D}$ and outputs $z \in \mathcal{R}$.

(Symmetric Key Encryption) SKE $:=$ (SKE.Enc, SKE.Dec) denotes the symmetric key encryption. SKE.Enc takes as input secret key $s k$ and plaintext $m$ and outputs ciphertext $c$. SKE.Dec decrypts the ciphertext $c$ using the same secret key $s k$ to generate plaintext $m$.

(Pseudorandom Function) PRF, PRF $: \mathcal{K}^{\prime} \times \mathcal{D}^{\prime} \rightarrow \mathcal{R}^{\prime}$ takes as input secret key $s k \in \mathcal{K}^{\prime}$ and message $m \in \mathcal{D}^{\prime}$ and provides an output which is indistinguishable from random.

(Fuzzy Extractor) FE := (FE.Gen, FE.Rec) denotes a fuzzy extractor. The FE.Gen algorithm takes as input a variable $z$ and outputs randomness $r$ and helper data $h d$. The FE.Rec algorithm recovers $r$ with input variable $z^{\prime}$ and $h d$ if $\operatorname{HD}\left(z, z^{\prime}\right)$ is sufficiently small. If $\operatorname{HD}\left(z, z^{\prime}\right) \leq d$ and $\bar{H}_{\infty}(z) \geq h$, the $(d, h)$-fuzzy extractor provides $r$ which is statistically close to random in $\{0,1\}^{|r|}$ even if $h d$ is exposed. The fuzzy extractor is usually constructed by combining an error-correction mechanism and a strong extractor.

\subsection{Parties and Trust Model}

We make assumptions comparable to earlier work in Authentication Protocols for constrained devices $[30,37,38]$. A trusted server and a set of num deployed devices will authenticate each other where devices require anonymous authentication. Before deployment, the devices are enrolled in a secure environment, using a one-time interface. After deployment, the server remains trusted, but the devices are subject to the actions of a malicious adversary (which is defined further).

Within this hostile environment, the server and the devices will authenticate each other such that the privacy of the devices is preserved against the adversary. The malicious adversary cannot determine the identity of the devices with a probability better than the security bound, and the adversary cannot trace the devices between different authentications.

The malicious adversary can control all communication between the server and (multiple) devices. Moreover, the adversary can obtain the authentication result from both parties and any data stored in the non-volatile memory of the devices. However, the adversary cannot mount implementation attacks against the devices, cannot reverse-engineer the PUF, nor can the adversary obtain any intermediate variables stored in registers or on-device RAM. We do not discount such attacks. For example, PUFs have been broken based on invasive analysis 
[31], side-channel analysis [11,35,32] and fault injection [12]. However, these attacks do not invalidate the protocol itself, and these attacks can be addressed with countermeasures at the level of the device.

\subsection{Secure and Privacy-preserving Authentication Protocol}

We propose a new authentication protocol by combining the privacy-preserving authentication protocol of Moriyama et al. [30] with the reverse fuzzy extractor mechanism of van Herrewege et al. [38].

The reverse fuzzy extractor works as follows [38]. The verifier sends a challenge $c$ to a PUF-enabled device. The device applies the challenge as input to a PUF, and obtains a noisy output $z^{\prime}$. The device then computes helper data $h d$ for this noisy output, and returns the helper data $h d$ and a hash of the output $z^{\prime}$ to the verifier. The verifier, who has previously enrolled the device, knows at least one output $z$ corresponding to the same challenge. The verifier can thus reconstruct $z^{\prime}$ using the helper data $h d$ and the previous output $z$. While this protocol moves the computationally expensive reconstruction phase to the verifier, the protocol does not maintain privacy. The device discloses its identity in order to allow the verifier to find a previous PUF output $z$.

Moriyama et al. proposed a PUF-based protocol that provides provably secure and private authentication [30]. Different from the existing PUF-based protocols, their protocol has a key updating mechanism that changes the shared secret key between the server and the device after each authentication. Furthermore, the secret key is derived from the PUF output. The Moriyama et al. protocol however places the PUF output reconstruction in the device.

The proposed protocol combines these two ideas into a merged protocol, illustrated in Fig. 1. We claim the same formal properties for the proposed protocol as for [30]. It works as follows. Each device is represented as a combination of a secret key $s k$ and a PUF challenge $y_{1}$. During secure initialization, the server initializes the secret key $s k_{1}$ in the device, and extracts the first PUF response $z_{1}$ from the device. The server keeps two copies of this information for each device in the database to support resynchronization. An authentication round proceeds as follows. First, the server sends a nonce to the device. The device extracts a first PUF output to construct an authentication field $c$ and a key $r_{1}$. The device then extracts a second PUF output $z_{2}^{\prime}$, which will be used during the next authentication round. The device encrypts this output (into $u_{1}$ ) and computes a MAC over it (into $v_{1}$ via PRF). The server will now try to authenticate the device. Initially, the server reconstructs the key $r_{1}$ using the reverse fuzzy extraction scheme. The server then performs an exhaustive search over the entire database in order to find a valid index. In case no match is found, the server will perform the same exhaustive search over the set of previous PUF outputs. If any match is found, the server will update its database to the next PUF output, and acknowledge the device. However, if both searches fail, the server will reply a random value. In the final step, the device verifies completion of authentication and updates its key tuple stored in non-volatile memory in case of acceptance.

The key features of the protocol can be summarized as follows. 


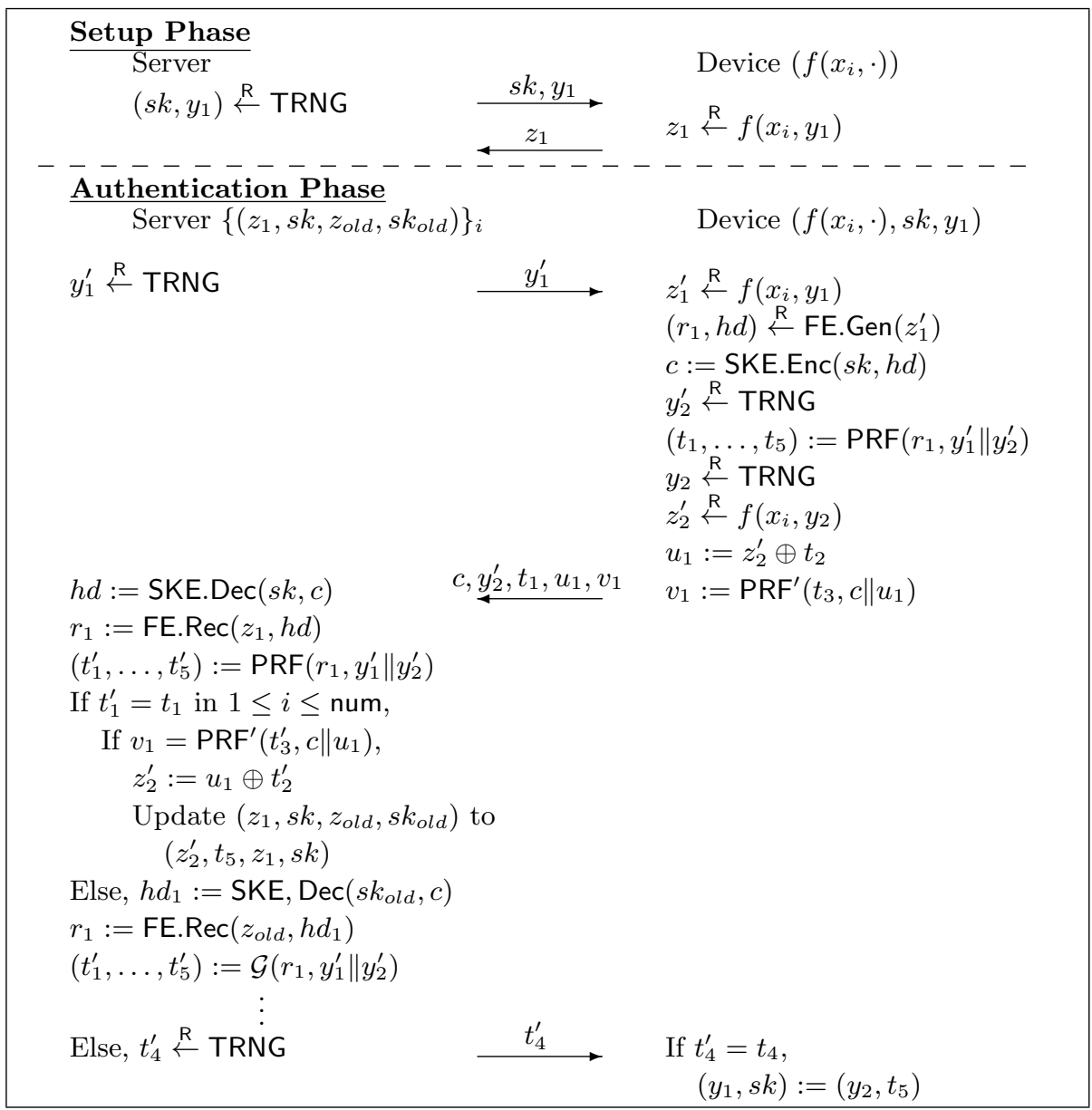

Fig. 1. The proposed PUF-based authentication protocol

Key Derivation via PUF with reverse FE. In the setup phase, the server stores the PUF output $z_{1}$ in the database. For each authentication, the device reads the PUF output $z_{1}^{\prime} \stackrel{R}{\leftarrow} f\left(x_{i}, y_{1}\right)$ with physical characteristic $x_{i}$ and generates helper data as $\left(r_{1}, h d\right) \stackrel{R}{\leftarrow} \mathrm{FE}$.Gen $\left(z_{1}^{\prime}\right)$. The helper data is encrypted and sent to the server as $c:=\operatorname{SKE}$.Enc $(s k, h d)$. The server decrypts it and executes verification with the shared secret $r_{1}:=\operatorname{FE} \cdot \operatorname{Rec}\left(z_{1}, h d\right)$.

Mutual Authentication and Authenticated Message Transmission. After deriving the shared secret $r_{1}$, the device and the server generate a random sequence $\left(t_{1}, \ldots, t_{5}\right) . t_{1}$ and $t_{4}$ are exchanged between the server and the device, and are used to implement mutual authentication. $t_{2}$ is used for XORed encryption of the PUF output, and $t_{3}$ is used as a secret key to generate va- 
lidity check value $v_{1} \cdot v_{1}$ serves as a MAC and prevents any modifications to the message $\left(c, u_{1}\right)$ since the server checks $v_{1}=\operatorname{PRF}^{\prime}\left(t_{3}^{\prime}, c \| u_{1}\right)$.

Key Update Mechanism. During the authentication, the device reads the PUF output twice, for different challenges. The second PUF output will be used to update the database if the authentication is successful. Upon verification of the device, the server updates the database with $\left(z_{2}^{\prime}, t_{5}\right)$. The last secret key $\left(z_{\text {old }}, s k_{\text {old }}\right)$ is still kept in the database and used for provision against the desynchronization attack. Even if $t_{4}^{\prime}$ is erased by an adversary, the reader can still trace and check the tag in the next protocol invocation.

Exhaustive Search. The device does not contain a fixed unique number of identity. Instead, the server launches an exhaustive search within the database to find an index $i \in\{1, \ldots$, num $\}$ which corresponds to the device. This authenticate-before-identify strategy [10] is a widely-known technique especially for anonymous lightweight authentication protocols (e.g., RFID authentication in [22]) to offer privacy. The search should execute in constant-time to avoid the abuse of a timing side-channel in a realistic usage. This is not hard to achieve but requires careful implementation of the server.

We have now identified the following protocol building blocks and demonstrate how to implement them in the next section.

- Physically unclonable function (e.g., $\left.z_{1}^{\prime} \stackrel{R}{\leftarrow} f\left(x_{i}, y_{1}\right)\right)$

- Random number generator (e.g., $y_{2}^{\prime} \stackrel{R}{\leftarrow}$ TRNG)

- Symmetric key encryption (e.g., $c:=\operatorname{SKE} . \operatorname{Enc}(s k, h d))$

- Pseudorandom function (e.g., $\left.\left(t_{1}, \ldots, t_{5}\right):=\mathcal{G}\left(r_{1}, y_{1}^{\prime} \| y_{2}^{\prime}\right)\right)$

- Fuzzy extractor (e.g., $\left(r_{1}, h d\right) \stackrel{R}{\leftarrow}$ FE.Gen $\left.\left(z_{1}^{\prime}\right)\right)$

\section{Instantiation of Protocol Components}

The protocol in the previous section assumes a generic security level. In this section, we discuss the instantiation of the main protocol components, assuming a security level of 128 bits. Our evaluation (Section 5) will show results for 64-bit as well as for 128-bit security.

\subsection{Architecture Assumptions}

Our prototype is implemented on a SASEBO-GII board. Besides the FPGA components, we make use of the on-board 2Mbit static RAM (ISSI IS61LP6432A) and a 16Mbit Flash (ATMEL AT45DB161D). The SRAM is organized as a $64 \mathrm{~K}$ memory with a 32-bit output. The Flash memory has an SPI (serial) interface. These component specifications are neither a requirement nor a limitation of our proposed design. Rather, we consider them pragmatic choices based on the available prototyping hardware. 


\subsection{Design of SRAM PUF}

The source of entropy in the design is an SRAM. We choose the SRAM for this role as the SRAM PUF is considered to be one of the most cost-efficient designs among recently proposed PUFs [27, Chapter 4]. It also offers reasonable noise levels. We are not aware of modeling attacks against SRAM PUF [34], and the known physical attacks against it are rather expensive [31, 17]. Furthermore, while we acknowledge the diversity of possible PUF designs for FPGA's [15, 1, 20, 24], the use of an SRAM PUF with simple power-cycling will yield a prototype that is less platform-specific. Our first step is to analyze the min-entropy, and the distribution of the startup values of the SRAM.

Min-entropy of SRAM. The min-entropy of the SRAM determines how many bytes of SRAM will be needed to construct one PUF output byte. We estimate the min-entropy of the SRAM empirically as follows. We collected the startup values of 90 SRAMs, collected from 90 different SASEBO-GII boards, each measured over 11 power cycles (990 x 2Mbit).

We then analyzed the Shannon Entropy as well as the min-entropy. Given a source of $n$ symbols with probabilities $b_{i}$, the efficiency of the source as measured in Shannon Entropy is computed as $\sum_{i=0}^{n}-b_{i} \log \left(b_{i}\right) / n \times 100$. At the bit-level, we found an efficiency of 34 to $46 \%$, depending on the board. This means that a bit on the average only holds between 0.34 and 0.46 bit of information, and indicates significant bias. We confirmed that there was bias according to the even and odd positions of the SRAM bytes.

We designed our PUF using the min-entropy, which is a worst-case metric. In this case, the min-entropy rate is computed as $n \times \min \left\{-b_{i} \log \left(b_{i}\right)\right\}_{i} \times 100$. When we analyzed the SRAM data at the byte level, we found a min-entropy of 5 to $15 \%$, which appeared to be caused by the abundance of the byte 0xaa at many SRAM locations. We did not investigate the cause of this bias, but we found that its effect can be considerably reduced by XORing adjacent bytes, and operation we will call 2-XOR. In this case the worst-case min-entropy rate becomes $26 \% 4$. We designed our PUF based on this value. In other words, we will use about 8 bytes of SRAM data to obtain one byte of entropy. The min-entropy estimate accounts for correlation between bits in a byte, which is more accurate than previous publications that used bit-level min-entropy estimates (e.g., $76 \%$ min-entropy rate in [8]).

Distribution of SRAM Data. A second important factor is the expected noise level for each SRAM, and the expected average Hamming distance between different SRAMs. We analyzed our data set over the different measurements per

\footnotetext{
${ }^{4}$ We select the maximum likelihood value within $0 \times 00-0 x F F$ in $2 \mathrm{Mbit}$ data and compute the min-entropy for each of the 990 datasets. Though the min-entropy rate we selected here, $26 \%$, is the lowest value in 990 datasets, our statistical tests showed that $95 \%$ of the datasets provide a min-entropy rate of $80 \%$ and a Shannon entropy rate of $98 \%$.
} 

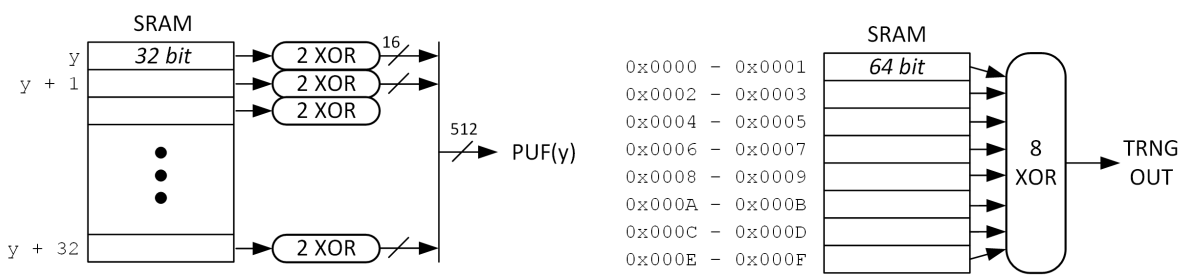

Fig. 2. (left) Design of the SRAM-PUF (right) Design of the SRAM-TRNG

SRAM. After applying the 2-XOR operation on the data, we found an average Hamming distance between same SRAM outputs of about 6.6 bit per word of 64 bit, which translates to a noise level of $10 \%$. When the SRAM outputs from different boards are compared, we found an average Hamming distance of 31.9 bit between words at the same address.

\subsection{Design of SRAM TRNG}

During authentication, the device requires a source of randomness. We reuse the SRAM as a random number generator, in order to minimize the device implementation cost. To obtain a noisy SRAM output, we XORed SRAM bytes multiple times. For each level of XORing, the noise level of the data is increased. We found that, after 8-fold XORing, the SRAM data passes all experiments in the NIST statistical Test Suite [36]. Hence, to generate a 128-bit random string from the device, we use 1024 bits of raw SRAM data. We can generate as much truly random data as there are available SRAM locations. One iteration of our protocol requires 652 random bits (see Table 1), which are extracted out of 5,216bit of SRAM data. Of course, the SRAM needs to be power-cycled after each iteration of the protocol.

Practical RAM organization. Figure 2 shows how the SRAM is used as a PUF and as a TRNG. In order to avoid direct correlation between PUF and TRNG data, we maintain separate address spaces for the PUF and the TRNG. In the prototype implementation, we allocate the first 256 SRAM words (of 32 bit each) for TRNG, while the remaining 65,280 words are used for the PUF. This means that the SRAM holds sufficient space for 2,040 PUF outputs $(2,040$ authentications). The input challenge to the PUF is therefore a 12-bit value $\mathrm{y}$, which is transformed into a base address for a block of 32 addresses by multiplying it with 32 and adding 0x100.

\subsection{Symmetric Key Encryption and PRF}

Our protocol requires a PRF and a symmetric-key encryption. We designed a PRF starting from the SIMON block cipher. It has the convenience that both 64-bit and 128-bit key size configurations are supported, and that very efficient implementations of it are known [4]. We select 128-bit block size for 128-bit 


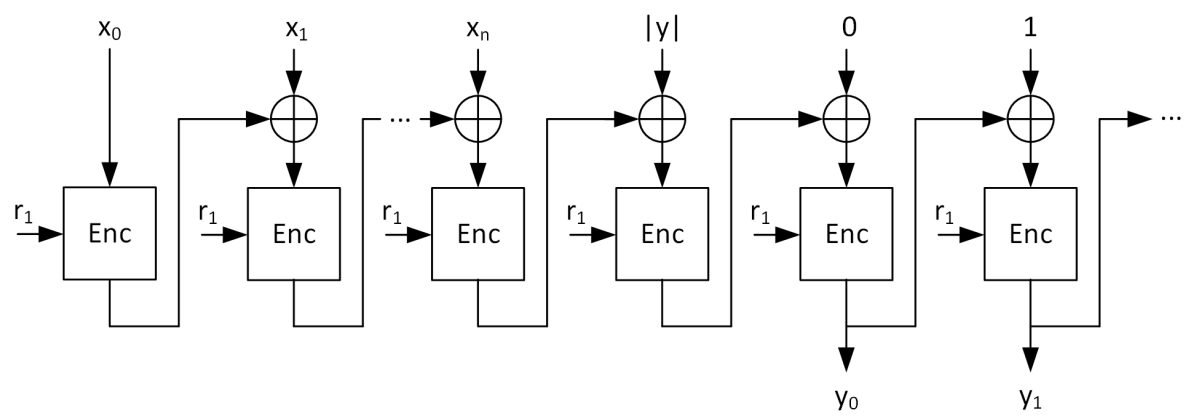

Fig. 3. PRF based on a block cipher in CBC mode. The variable-length message $x_{0}, . ., x_{n}$ is expanded using a secret $r_{1}$ into a message of length $|y|$

security. Using SIMON is neither a limitation nor a requirement of the prototype and it can be replaced with a secure symmetric-key cipher algorithm (e.g., AES) which supports the required security level.

Figure 3 shows how a PRF can be created using a block cipher in CBC mode. We assume SIMON does not provide any bias and the ciphertext is indistinguishable from random. An input message $x:=\left(x_{0}, \ldots, x_{n}\right)$ is encrypted with secret key $r_{1}$, then expanded into the output sequence $y:=\left(y_{0}, y_{1}, \ldots\right)$ by encrypting a counter value. The insertion of the output length parameter $|y|$ ensures that, even when the input and secret is identical, the PRF produces independent output sequences when the specified output size is different.

\subsection{Design of Fuzzy Extractor}

In this section, we describe the design of the fuzzy extractor, including the error correction and the strong extractor.

Error Correction. Various techniques for error correction have been proposed in recent years, with mechanisms based on code-offset [13], index-based syndrome coding [39], and pattern matching [33]. We adopt the following code-offset mechanism using a $\mathrm{BCH}\left(n_{1}, k_{1}, d_{1}\right)$ code [13]. The code allows to correct errors up to $\left\lfloor\left(d_{1}-1\right) / 2\right\rfloor$-bit within a $n_{1}$-bit block. Two procedures, BCH.Gen and BCH.Dec, represent encoding and decoding respectively:

Encode $(a): \delta \stackrel{R}{\leftarrow} \operatorname{TRNG} \in\{0,1\}^{k_{1}}, c w:=\mathrm{BCH} . \operatorname{Gen}(\delta) \in\{0,1\}^{n_{1}}, h d:=a \oplus c w$

$\operatorname{Decode}\left(a^{\prime}, h d\right): c w^{\prime}:=a^{\prime} \oplus h d, c w:=\operatorname{BCH} \cdot \operatorname{Dec}\left(c w^{\prime}\right), a:=c w \oplus h d$

The PUF output $a$ is XORed with a random codeword $c w$ to construct $h d$. While $h d$ is not secret, the PUF output $a$ must remain secret. We consider the complexity of finding $a$. For a single block, this complexity is $2^{k_{1}}$. For a PUF output $z_{1}$ mapped into multiple $n_{1}$-bit blocks, the complexity is $2^{k_{1} \cdot\left|z_{1}\right| / n_{1}}$. It should be higher than the selected security level of 128 bit.

We use 504 bits of a 512-bit PUF output in 8 blocks of a $\operatorname{BCH}(63,16,23)$ code, which gives us the desired security level. The $\mathrm{BCH}(63,16,23)$ code corrects 

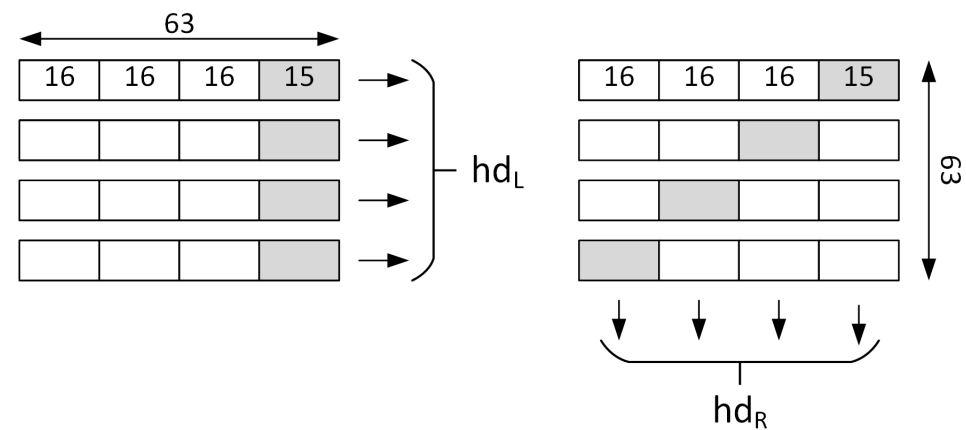

Fig. 4. Helper data construction. A 252-bit field is split into 463 -bit blocks and encoded as $h d_{L}$. Next, each block is left-rotated over $0,16,32$ and 48 bits respectively. Finally, 463 -bit columns are encoded to produce $h d_{R}$. A 504-bit field (needed for the 128-bit security level) is encoded by applying this construction twice.

up to $17.5 \%$ noisy bits, which appears to be above the observed SRAM noise level of $10.0 \%$. However, this is too optimistic. If we assume that a single bit flips with a probability of $10.0 \%$, then there is a $2.36 \%$ probability that 12 bits or more will flip in a 63-bit block, and thus produce a non-correctable error. This translates to a probability of only $(1-0.0236)^{8} \times 100 \approx 82.6 \%$ that 8 blocks of a 504-bit PUF output can be fully corrected. Therefore, we need a better error correction mechanism.

We apply an interleaved coding technique as illustrated in Figure 4. A 252-bit data field is organized as a matrix with fields of $\{16,16,16,15\}$ bits per row. The encoding of each 63 -bit row yields helper data $h d_{L}$. Next, each row of the matrix is rotated over a multiple of 16-bits, such that 63 -bit columns are obtained. The encoding of the columns now yields helper data $h d_{R}$. The overall helper data is $h d_{L}|| h d_{R}$. To encode a 504-bit field, we apply this construction twice. Compared an earlier interleaved-coding design by Gassend [14], our technique accommodates odd-sized rows and columns.

Error decoding is performed adaptively. We first correct the rows, then decode remaining faulty bits over the columns. Figure 5 plots the probability of a faulty output after the error decoding as a function of the error probability of the PUF output. The residual error rate is $1-1.92 \times 10^{-6}$, which is comparable to the acceptable error rate for standard performance levels in [27]. Several authors have proposed techniques to improve the reliability of SRAM PUF with respect to environmental conditions and aging $[9,28]$. These techniques, when applied to our design, may allow to reduce the complexity of the error correction code.

Security Analysis. The computational complexity to find 252-bit PUF data from the helper data is $2^{64}$. The helper data over the rows $h d_{L}$ and columns $h d_{R}$ are generated using independent random code-words $c w_{L}$ and $c w_{R}$, respectively. The BCH encoding function expands the randomness of a 16-bit seed into a 63-bit codeword. The method ensures that XOR combinations of $h d_{L}$ and $h d_{R}$ 


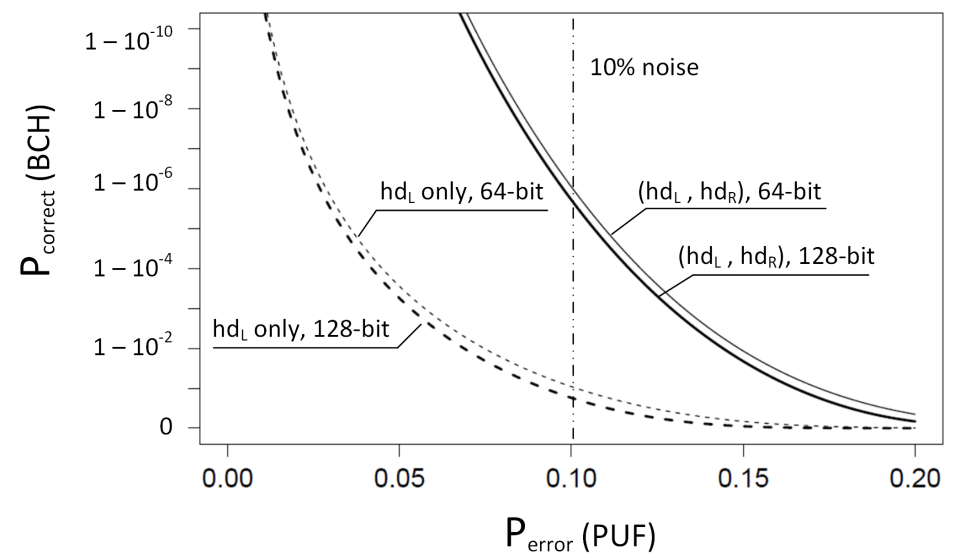

Fig. 5. Probability for a faulty PUF output using the proposed interleaved coding technique.

do not explicitly leak PUF data, and it employs the working heuristic that these combinations are 'random enough'. We experimentally verified that the $2^{16}$ possible $\mathrm{BCH}$ code words, parsed into $\{16,16,16,15\}$-bit fields, show no collisions within a field. Thus $2^{16}$ us the security level per code word ${ }^{5}$. The entire matrix is covered by four independent code words over the rows, and four independent code words over the columns. An attack of $2^{64}$ complexity, is to guess four code words and then use the helper data to estimate the PUF output. Since every element of the matrix holds different PUF output bits, the adversary must find at least the code words over all the rows, or the code words over the all columns. That is a lower bound for this attack strategy, because four codewords over a combination of rows and columns cannot cover the complete matrix, and therefore cannot recover all PUF output bits. As noted above, the dependency $h d_{L} \oplus c w_{L}=h d_{R} \oplus c w_{R}$, cannot reduce the complexity of the search below $2^{64}$, since every single code word has security level $2^{16}$, and since the smallest number of code-words required to recover the PUF output data is four ${ }^{6}$.

Strong Extractor. The role of strong extractor is to reduce the non-uniform data (PUF output data) to the required entropy level. We assume the proposed PRF works as a strong extractor. As discussed earlier, the PRF still uses a

${ }^{5}$ One may think that the helper data from a $(63,16,23)-\mathrm{BCH}$ code decreases the min-entropy by $63-16=47$-bit. This means that the PUF's data must always have full-entropy (i.e., min-entropy rate is 1 ) from the view point of information theory. Instead, we evaluate the computational complexity that the adversary can really guess the PUF's original data by observing the helper data.

${ }^{6}$ Recently, Becker suggested that this interleaved construction should be improved to avoid linear equation analysis. Since each bit of the helper data is derived by the code-offset construction, an adversary can obtain 504 equations whereas the number of unknown variables are $252+8 \times 16=380$ [5]. 
secret key. The secret key $s k^{\prime}$ is pre-shared and updated after every successful authentication. The strong extractor is a probabilistic function, and requires a random input rnd. Following Håstad et al. [16], we select the size of rnd to be twice the security level. For 128-bit security, $\mid$ rnd $\mid=256$ is sufficient to derive 128-bit randomness with input 128-bit min-entropy data (i.e. 504-bit PUF's output $z_{1}^{\prime}$ ).

\subsection{Relevant Data Sizes and Key Lengths in Protocol}

From the above analysis and instantiation, we summarize the length for each variable for 64-bit and 128-bit security in Table 1.

Table 1. Key Length and Data Sizes (in bits) for the proposed protocol

\begin{tabular}{|l|l|l|r|r|}
\hline Category & Purpose & Variables & $\begin{array}{r}\text { 64-bit } \\
\text { security }\end{array}$ & $\begin{array}{r}\text { 128-bit } \\
\text { security }\end{array}$ \\
\hline Setup Phase & Input address & 12 & 12 \\
& PUF's output & $y_{1}$ & 252 & 504 \\
& Stored key & $z_{1}$ & 64 & 128 \\
\hline \hline Authentication & PUF's output $s k^{\prime}$ & 252 & 504 \\
Phase & Nonce & $z_{1}^{\prime}, z_{2}^{\prime}$ & 64 & 128 \\
& Randonmess for FE & $y_{1}^{\prime}, y_{2}^{\prime}$ & 128 & 256 \\
& Secret key for PRF & $\delta$, rnd & 64 & 128 \\
& Helper data & $h d$ (includes rnd) & 632 & 1,264 \\
& Ciphertext & $c$ & 640 & 1,280 \\
& PUF's input & $y_{1}$ & 12 \\
& Mutual authentication & $y_{1}, t_{4}$ & 64 & 128 \\
& XORed element & $t_{2}$ & 252 & 504 \\
& Secret key for PRF ${ }^{\prime}$ and MAC & $t_{3}, s_{1}$ & 64 & 128 \\
& Updated stored key & $t_{5}$ & 128 & 256 \\
\hline \hline Communication & First message (from server) & $y_{1}^{\prime}$ & 64 & 128 \\
& Second message (from device) & $\left(c, y_{2}^{\prime}, t_{1}, u_{1}, s_{1}\right)$ & 1,084 & 2,168 \\
& Third message (from server) & $t_{4}^{\prime}$ & 64 & 128 \\
\hline \hline Memory & Persistent State (NVM) & $\left(s k, s k^{\prime}, y_{1}\right)$ & 140 & 268 \\
& SRAM area for PUF & & 504 & 1,008 \\
& SRAM area for RNG & & 2,656 & 5,216 \\
\hline
\end{tabular}

\section{Architecture Design}

In this section, we describe the architecture design of the implementation. We introduce the overall design, discuss the detailed implementation of the cryptographic accelerator, and finally discuss the prototype evaluation.

\subsection{System Design}

Figure 6 illustrates the system architecture with the device and the server. They are emulated with a SASEBO-GII board and a PC respectively. The basis of the 


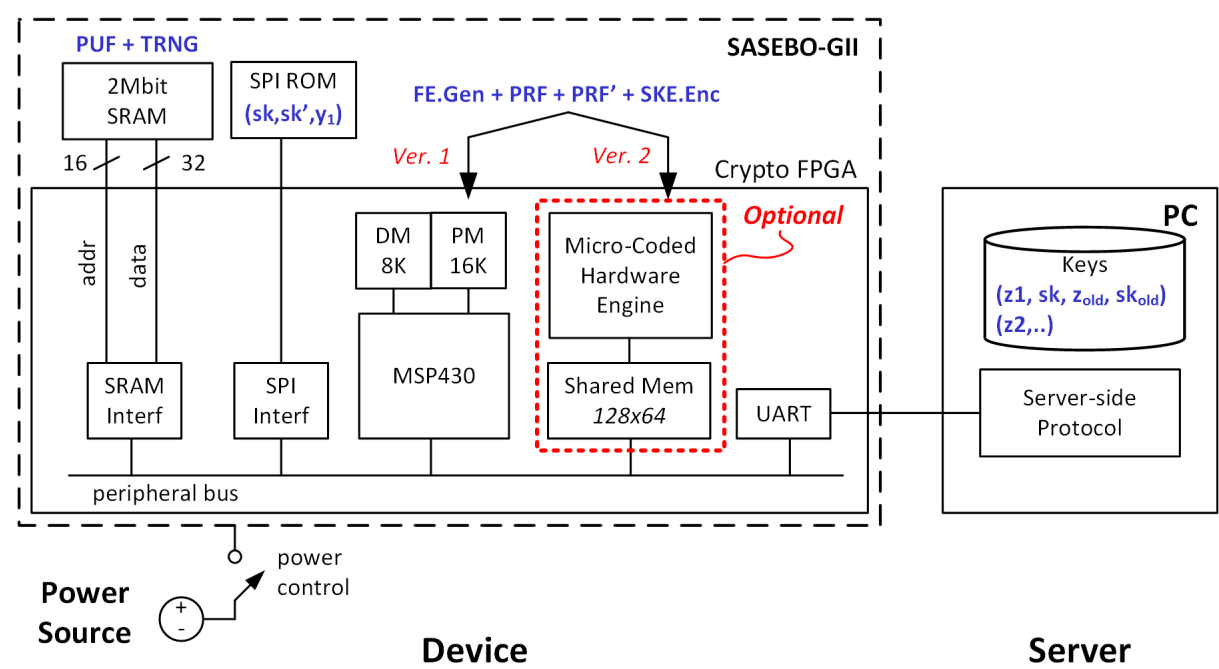

Fig. 6. System Architecture of the Device and Server

device is an MSP430 Microcontroller mapped as a soft-core into the Crypto FPGA of the SASEBO-GII board. The design integrates an SRAM, a nonvolatile memory, a UART, and optionally a hardware accelerator. The MSP430 core has its own program memory and data memory; the SRAM is used solely as a source of entropy. The power source to the device is controlled as part of the testing environment.

The server manages a database with secret keys and PUF responses. For each device authenticated through this server, the database stores two pairs of keys and PUF responses, one for the current authentication $\left(z_{1}, s k\right)$, and one from the previous authentication $\left(z_{\text {old }}, s k_{\text {old }}\right)$. The communication between the device and the server is implemented through a serial connection.

The 16-bit MSP430 microcontroller is configured with 8KByte of data memory and $16 \mathrm{KByte}$ of program memory. We will discuss the detailed memory requirements of the protocol in Section 5. We implement two different versions of this design. In the first version, the protocol is mapped fully in $\mathrm{C}$ and executed on the MSP430. In the second version, the major computational bottlenecks, including Fuzzy Extractor Generation (FE.Gen), PRF computation (PRF and $\mathrm{PRF}^{\prime}$ ) and Encryption (SKE.Enc) are executed in the hardware engine. In this configuration, the MSP430 is used as a data multiplexer between the UART, the SRAM, the non-volatile memory and the hardware engine.

Protocol Mapping and Execution. The protocol includes a single setup phase, followed by one or more authentication phases. Before the execution of each phase, we power-cycle the device to re-initialize the SRAM PUF. This gives us a real SRAM PUF noise profile. Table 2 shows a detailed description of the protocol authentication phase on the architecture of Figure 6 . The operations are 
shown for the software-only implementation (Ver. 1) as well as for the hardwareengine enabled implementation (Ver. 2). Table 2 demonstrates the principal data flows in the architecture. For example, "SPIROM. Read $\rightarrow$ MSP430.DM" means that data is copied from the SPI-ROM to the MSP430 data memory.

Table 2. Principal Data Flows during Execution of the Authentication Protocol on the Device. Dataflow notation A.a $\rightarrow$ B.b indicates that data from A (port/method a) is forwarded to B (port/method b)

\begin{tabular}{|c|c|c|c|}
\hline Seq & Authen & $\begin{array}{c}\text { MSP430 } \\
\text { Fig. } 6 \text { Ver. } 1\end{array}$ & $\begin{array}{c}\text { MSP } 430+\mathrm{HW} \text { Engine } \\
\text { Fig. } 6 \text { Ver. } 2\end{array}$ \\
\hline 1 & 91 & UART . Receive $\rightarrow$ MSP43 & UART.Receive $\rightarrow$ MSP430.D \\
\hline 2 & Read $s k, s k^{\prime}, y_{1}$ & SPIROM.Read $\rightarrow$ MSP4 & SPIROM.Read $\rightarrow$ MSP430.DM \\
\hline 3 & $z_{1}^{\prime} \stackrel{\mathrm{R}}{\leftarrow} f\left(x_{i}, y_{1}\right)$ & SRAM.PUF $\rightarrow$ MSP430.DM & SRAM.PUF $\rightarrow$ MSP430.DM \\
\hline 4 & $\left(r_{1}, h d_{1}\right) \stackrel{\mathrm{R}}{\leftarrow} \mathrm{FE} \cdot \operatorname{Gen}\left(z_{1}^{\prime}\right)$ & MS430 $\operatorname{run}(\mathrm{PRF})$ & \\
\hline & $m_{2} \stackrel{R}{\leftarrow}$ TRNG & SRAM TRNC $\longrightarrow$ MSP 430 DM & \\
\hline & $y_{2} \stackrel{R}{\leftarrow}$ TRNG & SRAM.TRNG $\rightarrow$ MSP430.DM & SRAM.TRNG $\rightarrow$ MSP430.DM \\
\hline 6 & $\left(t_{1}, \ldots\right.$ & an (PRF) & \\
\hline 7 & $c:=\operatorname{SKE} . \operatorname{Enc}\left(s k, h d_{1}\right)$ & an (Enc) & \\
\hline 8 & $z_{2}^{\prime} \stackrel{\mathrm{R}}{\leftarrow} f\left(x_{i}, y_{2}\right)$ & $\mathrm{UF} \rightarrow \mathrm{MSP} 430 . \mathrm{DM}$ & SRAM.PUF $\rightarrow$ MSP430.DM \\
\hline 9 & $u_{1}:=z_{2}^{\prime} \oplus t_{2}$ & $\operatorname{run}(\mathrm{x} o r)$ & \\
\hline 10 & $v_{1}:=\operatorname{PRF}^{\prime}\left(t_{3}\right.$, & $\operatorname{run}(\mathrm{PRF})$ & \\
\hline 11 & ecution & & $\begin{array}{l}\text { MSP } 430 . \text { DM } \rightarrow \text { HW. SharedMem } \\
\text { HW.run } \\
\text { HW.SharedMem } \rightarrow \text { MS430.DM }\end{array}$ \\
\hline 12 & $\imath_{2}, t_{1}$ & UART & MSP430.DM $\rightarrow$ UART.Send \\
\hline 13 & $t_{4}^{\prime}$ & $\mathrm{e} \rightarrow \mathrm{MS}$ & UART.Receive $\rightarrow$ MSP430.D \\
\hline 14 & Write $y_{2}, t_{5}$ & MSP430.DM $\rightarrow$ SPIROM.Write & MSP430.DM $\rightarrow$ SPIROM. Write \\
\hline
\end{tabular}

Hardware Engine Integration. The communication between the microcontroller and the hardware engine is implemented through a shared-memory. The microcontroller initializes the input arguments for the hardware engine in the shared memory, initiates the protocol computation, and waits until a completion notification of the hardware engine. After completion, the result of the computation is available in the shared memory. Furthermore, a single execution on the hardware engine takes multiple steps in the protocol: PRF computation, $\mathrm{BCH}$ Encoding, and SIMON encryption. When the hardware engine is used, the arguments are first collected in the MSP430 data memory, before they are copied to the shared memory (Table 2 step 11). There is some overhead introduced because of this particular design, but we will show that the resulting implementation still significantly outperforms a software-only design. 


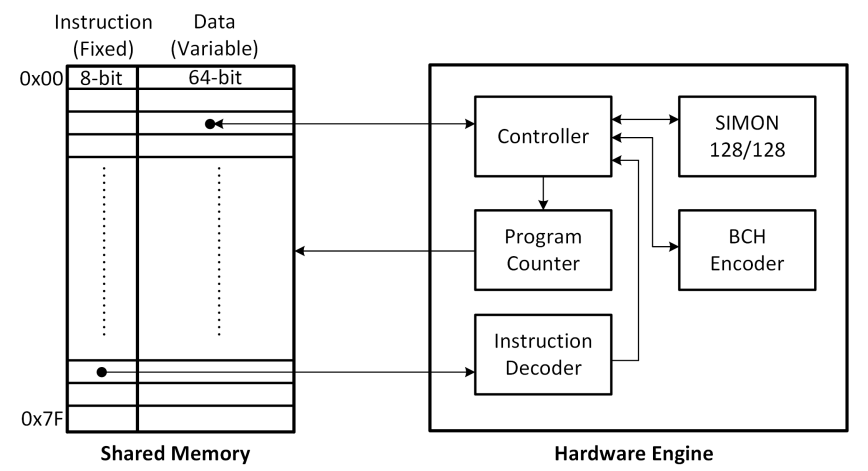

Fig. 7. Block Diagram of the Hardware Engine

\subsection{Hardware Engine}

The purpose of the hardware engine is to accelerate the PRF computation, $\mathrm{BCH}$ encoding, and SIMON encryption. Indeed, our profiling results (discussed further, Table 5) show that these operations constitute to $88 \%$ of the total execution time. The protocol can be realized with a small and fixed microprogram so we applied a micro-coded design methodology. Moreover, since it is efficient to use a RAM to store the protocol variables, the very same memory can also store the micro-coded instructions. Although this design is prototyped on FPGAs, it can also target dedicated hardware. By changing the microprogram, we can extend this architecture to other protocols as well.

Figure 7 shows the block diagram of the hardware engine. It uses the roundserial version of SIMON 128/128 for the PRF and encryption operations, and an LFSR-based implementation of the BCH encoding for the error correction part of the FE.Gen. Therefore, it takes 68 clock cycles to encrypt one 128-bit block and 16 clock cycles to encode one 16-bit block.

The shared memory between the MSP430 and the micro-coded hardware engine is a single memory element which has a word size of 72-bits. The least significant 64-bits of each word store the data, while the most significant 8-bits store the micro-coded instruction. Since these instructions are fixed at design time, this section of the memory is treated as a ROM. After the hardware engine reads a word from the memory, it decodes the micro-coded instruction. Then based on the decoded value, the controller selects which operation to run with the associated data and updates the value of the program counter.

\section{Evaluation}

In this section, we first discuss the device implementation cost, and then evaluate the system performance of our protocol. We implemented three different device configurations, including the 64-bit and 128-bit security level of the softwareonly implementation (Fig. 6 Ver. 1), as well as the 128-bit security level of the hardware-engine enabled implementation (Fig. 6 Ver. 2). 
Table 3. MSP430 Memory Footprint. Data area includes Global and Local Variables (stack, bss and data).

\begin{tabular}{|c|c|c|c|c|c|}
\hline \multicolumn{2}{|r|}{ Category } & $\begin{array}{l}\text { 64-bit } \\
\text { MSP430 }\end{array}$ & $\begin{array}{l}\text { 128-bit } \\
\text { MSP430 }\end{array}$ & $\begin{array}{c}\text { 128-bit } \\
\text { HW engine } \\
+ \\
\text { MSP430 }\end{array}$ & Unit \\
\hline \multirow{7}{*}{ Text } & HW Abstraction & 1,022 & 1,022 & $\overline{1,398}$ & bytes \\
\hline & Communications & 496 & 644 & 628 & bytes \\
\hline & SIMON PRF & 1,604 & 2,440 & 0 & bytes \\
\hline & BCH Encoding & 1,214 & 1,214 & 0 & bytes \\
\hline & PUF + Fuzzy Extr & 562 & 646 & 590 & bytes \\
\hline & TRNG & 396 & 456 & 396 & bytes \\
\hline & Protocol & 1,568 & 1,682 & 1,908 & bytes \\
\hline \multicolumn{2}{|c|}{ Overall Text } & 6,862 & 8,104 & 4,920 & bytes \\
\hline \multirow{2}{*}{ Data } & Variables & 424 & 656 & 656 & bytes \\
\hline & Constants & 197 & 197 & 73 & bytes \\
\hline \multicolumn{2}{|c|}{ Overall Data } & 621 & 853 & 729 & bytes \\
\hline
\end{tabular}

Table 4. Hardware Utilization (Xilinx XC5VLX30-1FFG324 System Clock 1.846 MHz)

\begin{tabular}{|l|r|r|r|}
\hline \multicolumn{1}{|c|}{ Module } & \multicolumn{1}{|c|}{ LUT } & Registers & Block RAM \\
\hline MSP430 Core & 2084 & 684 & 4 \\
MSP430 Program Mem & & & \\
MSP430 Data Mem & & & \\
SRAM Interface & 54 & 30 & \\
SPI ROM Interface & 45 & 30 & \\
UART & 139 & 106 & \\
\hline HW Engine & 1221 & 441 & \\
HW Shared Mem & & & \\
\hline Overall & 3543 & 1275 & \\
\hline
\end{tabular}

\subsection{Implementation Cost}

Table 3 shows the memory footprint required for each version, including the size of the MSP430 object code, and the data-memory requirements. We used the GNU gcc version 4.6.3 to compile C for the MSP430 at optimization level 2. As our main objective was to demonstrate the implementation of the complete protocol, we did not use low-level programming techniques. However, the data indicates that the protocol already fits into a small microcontroller. When the hardware engine is enabled, the tasks of the MSP430 reduce to interfacing the SRAM, NVM and UART. We envisage that it is feasible to completely remove the MSP430 microcontroller by having the hardware engine directly access these peripherals.

Table 4 lists the hardware requirements for the baseline design, which is shared among all versions of the protocol. The hardware engine is about half as big as the MSP430 core. 
Table 5. Implementation Performance in System Clock Cycles.

\begin{tabular}{|c|c|c|c|c|}
\hline Protocol Step & $\begin{array}{c}\text { Implementation } \\
\text { Target }\end{array}$ & $\begin{array}{c}\text { 64-bit } \\
\text { Fig. } 6 \text { Ver. } 1 \\
\end{array}$ & $\begin{array}{c}\text { 128-bit } \\
\text { Fig. } 6 \text { Ver. } 1 \\
\end{array}$ & $\begin{array}{l}\text { 128-bit w. } \\
\text { HW Engine } \\
\text { Fig. } 6 \text { Ver. } 2 \\
\end{array}$ \\
\hline Read $s k, s k^{\prime}, y_{1}$ & Read Rc & 31,356 & 61,646 & 61,646 \\
\hline$y_{2}^{\prime} \stackrel{R}{\leftarrow}$ TRNG, $y_{2} \stackrel{R}{\leftarrow}$ TRNG & SRAM TRNG & 11,552 & 23,341 & 22,981 \\
\hline$z_{1}^{\prime} \stackrel{R}{\leftarrow} f\left(x_{i}, y_{1}\right), z_{2}^{\prime} \stackrel{R}{\leftarrow} f\left(x_{i}, y_{2}\right)$ & SRAM PUF & 4,384 & 9,082 & 8,741 \\
\hline$\left(r_{1}, h d\right) \stackrel{\mathrm{R}}{\leftarrow} \mathrm{FE} \cdot \operatorname{Gen}\left(z_{1}^{\prime}\right)$ & $\begin{array}{l}\text { BCH Encoder } \\
\text { Strong Extractc }\end{array}$ & $\begin{array}{r}268,820 \\
28,691\end{array}$ & $\begin{array}{l}485,094 \\
205,080\end{array}$ & \multirow{4}{*}{18,597} \\
\hline$\left(t_{1}, \ldots, t_{5}\right):=\operatorname{PRF}\left(r_{1}, y_{1}^{\prime} \| y_{2}^{\prime}\right)$ & PRF & 44,355 & 299,724 & \\
\hline$c:=\operatorname{SKE} . \operatorname{Enc}(s k, h d)$ & Encryption & 39,583 & 252,829 & \\
\hline$v_{1}:=\operatorname{PRF}^{\prime}\left(t_{3}, c \| u_{1}\right)$ & PRF' & 57,601 & 394,126 & \\
\hline Overall & & 486,343 & $1,730,922$ & 111,965 \\
\hline Write $y_{2}, t_{5}$ & Write ROM (SPI) & $\overline{776,290}$ & 128,829 & $\overline{128,849}$ \\
\hline
\end{tabular}

\subsection{Performance}

Table 5 lists the performance of our design, measured in system clock cycles. We implemented this design at a System Clock of $1.846 \mathrm{Mhz}$ to reflect the constrained platform for the device. The hardware engine can drastically reduce the cycle count of the implementation. The cycle count shown for the hardware engine includes the overhead of preparing data; the actual compute time is only 4,486 cycles.

\subsection{Related Work}

The comparison of this design to related works is not obvious because previous publications did not implement an end-to-end demonstrator. Table 6 presents a comparison of related realizations. We emphasize our design has many advantages (such as flexibility, formal properties, full implementation) that cannot be expressed as a single quantity.

\subsection{Benchmark Analysis}

We analyzed our protocol with respect to a recently published benchmark for PUF based protocols [10]. Our protocol is implemented using a weak PUF. The protocol requires $n+1$ challenge-response pairs for $n$ authentications. The total number of PUF responses depends on the anonymity needs of the application.

The protocol supports server authenticity, device authenticity, device privacy, and leakage resilience. It can use $d$-enrollments for a perfect privacy use-case and $(\infty)$-enrollments without token anonymity. The system is noise-robust and modelling-robust. Mutual authentication provides both server and user authenticity. Moreover, since the protocol does not have an internal synchronization, it is not susceptible to DoS attacks. Our protocol enables token privacy and the security proof confirms leakage resilience. 
Table 6. Comparison with previous work

\begin{tabular}{|c|c|c|c|c|}
\hline Reference & PUFKY [27] & Slender $[29]$ & Reverse-FE [38] & This work \\
\hline Operation & Key generation & Protocol & Protocol & Protocol \\
\hline Privacy & No & No & No & Yes \\
\hline Security flaws & No & Major [10] & Minor [10] & No \\
\hline $\begin{array}{l}\text { Implemented } \\
\text { Parties }\end{array}$ & $\mathrm{N} / \mathrm{A}$ & Device & Device & $\begin{array}{l}\text { Device, } \\
\text { Server }\end{array}$ \\
\hline $\begin{array}{l}\text { Communication } \\
\text { Interface }\end{array}$ & Yes: Bus & No & No & Yes: Bus, UART \\
\hline Flexibility & Low & Low & Low & High \\
\hline $\begin{array}{l}\text { Reconfiguration } \\
\text { Method }\end{array}$ & $\begin{array}{l}\text { Redesign } \\
\text { Hardware }\end{array}$ & $\begin{array}{l}\text { Redesign } \\
\text { Hardware }\end{array}$ & $\begin{array}{l}\text { Redesign } \\
\text { Hardware }\end{array}$ & $\begin{array}{c}\text { Modify Software, } \\
\text { Update Microcode }\end{array}$ \\
\hline Demonstrator & FPGA & FPGA & FPGA & $\mathrm{FPGA}+\mathrm{PC}$ \\
\hline Security-level & 128-bits & 128-bits & 128-bits & 64,128-bits \\
\hline $\begin{array}{l}\text { Execution time } \\
\text { (clock cycles) }\end{array}$ & 55,310 & - & - & 18,597 \\
\hline $\begin{array}{l}\text { Logic Cost } \\
\text { (w/o PUF) }\end{array}$ & 210 Slices & $\begin{array}{c}144 \text { LUT, } \\
274 \text { Register }\end{array}$ & $\begin{array}{l}658 \text { LUT, } \\
\text { 496 Register }\end{array}$ & $\begin{array}{l}1221 \text { LUT, } \\
\text { 441 Register }\end{array}$ \\
\hline PUF-type & Strong-PUF & Strong-PUF & - & Weak-PUF \\
\hline PUF-instance & RO-PUF & XOR-Arbiter & - & SRAM \\
\hline Hardware Platform & XC6SLX45 & XC5VLX110T & XC5VLX50 & XC5VLX30 \\
\hline
\end{tabular}

\section{Conclusion}

We demonstrated the challenging path from the world of protocol theory to concrete software/hardware realization for the case of a privacy preserving authentication protocol. We observe that bringing all components of a protocol together in a single embodiment is a vital and important step to check its feasibility. Furthermore, the formal basis of the protocol is crucial to prevent cutting corners in the implementation.

Even though we claim this work is the first demonstration of a PUF-based protocol with a formal basis, there is always room for improvement. First, the current implementation can be optimized at the architectural level, for throughput, area, or power [3]. Second, new components and algorithms, such as novel PUF architectures [19] or novel coding techniques [18], may enable us to revisit steps within the protocol itself.

Acknowledgements: The project was supported in part by the National Science Foundation Grant 1314598 and 1115839. Part of the work of M. Yung was done when visiting the Simons Institute for Theory of Computing, U.C. Berkeley. The authors thank the anonymous reviewers for their comments, discussions with Mandel Yu and useful comments from Georg T. Becker. 


\section{References}

1. Anderson, J.H.: A PUF design for secure FPGA-based embedded systems. In: ASP-DAC 2010. pp. 1-6. IEEE (2010)

2. Aysu, A., Gulcan, E., Moriyama, D., Schaumont, P., Yung, M.: End-to-end design of a PUF-based privacy preserving authentication protocol. In: Güneysu, T., Handschuh, H. (eds.) CHES 2015. LNCS, vol. 9293, pp. 556-576. Springer, Heidelberg (2015)

3. Aysu, A., Gulcan, E., Schaumont, P.: SIMON says: Break area records of block ciphers on FPGAs. Embedded Systems Letters 6(2), 37-40 (2014)

4. Beaulieu, R., Shors, D., Smith, J., Treatman-Clark, S., Weeks, B., Wingers, L.: The SIMON and SPECK families of lightweight block ciphers. IACR Cryptology ePrint Archive 2013, 404 (2013)

5. Becker, G.T.: Personal communication (2015)

6. Bernstein, D.J.: Error-prone Cryptographic Designs. Real World Cryptography Workshop (January 2015), http://cr.yp.to/talks/2015.01.07/ slides-djb-20150107-a4.pdf

7. Bolotnyy, L., Robins, G.: Physically unclonable function-based security and privacy in RFID systems. In: PerCom 2007. pp. 211-220. IEEE (2007)

8. Claes, M., van der Leest, V., Braeken, A.: Comparison of SRAM and FF PUF in 65nm technology. In: Laud, P. (ed.) NordSec 2011. LNCS, vol. 7161, pp. 47-64. Springer, Heidelberg (2011)

9. Cortez, M., Hamdioui, S., van der Leest, V., Maes, R., Schrijen, G.J.: Adapting voltage ramp-up time for temperature noise reduction on memory-based PUFs. In: HOST 2013. pp. 35-40. IEEE (2013)

10. Delvaux, J., Gu, D., Peeters, R., Verbauwhede, I.: A survey on lightweight entity authentication with strong PUFs. IACR Cryptology ePrint Archive 2014, 977 (2014), http://eprint.iacr.org/2014/977

11. Delvaux, J., Verbauwhede, I.: Side channel modeling attacks on $65 \mathrm{~nm}$ arbiter PUFs exploiting CMOS device noise. In: HOST 2013. pp. 137-142. IEEE (2013)

12. Delvaux, J., Verbauwhede, I.: Fault injection modeling attacks on $65 \mathrm{~nm}$ arbiter and RO sum PUFs via environmental changes. IEEE Trans. on Circuits and Systems 61-I(6), 1701-1713 (2014)

13. Dodis, Y., Ostrovsky, R., Reyzin, L., Smith, A.: Fuzzy extractors: How to generate strong keys from biometrics and other noisy data. SIAM J. Comput. 38(1), 97-139 (2008)

14. Gassend, B.: Physical Random Fuctions. Master's thesis, Massachusetts Institute of Technology (2003)

15. Güneysu, T.: Using data contention in dual-ported memories for security applications. Signal Processing Systems 67(1), 15-29 (2012)

16. Håstad, J., Impagliazzo, R., Levin, L.A., Luby, M.: A pseudorandom generator from any one-way function. SIAM J. Comput. 28(4), 1364-1396 (1999)

17. Helfmeier, C., Boit, C., Nedospasov, D., Seifert, J.: Cloning physically unclonable functions. In: HOST 2013. pp. 1-6. IEEE (2013)

18. Herder, C., Ren, L., van Dijk, M., Yu, M.M., Devadas, S.: Trapdoor computational fuzzy extractors. IACR Cryptology ePrint Archive 2014, 938 (2014), http://eprint.iacr.org/2014/938

19. Holcomb, D.E., Fu, K.: Bitline PUF: building native challenge-response PUF capability into any SRAM. In: Batina, L., Robshaw, M. (eds.) CHES 2014, LNCS, vol. 8731, pp. 510-526. Springer, Heidelberg (2014) 
20. Hori, Y., Kang, H., Katashita, T., Satoh, A., Kawamura, S., Kobara, K.: Evaluation of physical unclonable functions for 28-nm process field-programmable gate arrays. JIP 22(2), 344-356 (2014)

21. Jin, Y., Xin, W., Sun, H., Chen, Z.: PUF-based RFID authentication protocol against secret key leakage. In: Sheng, Q.Z., Wang, G., Jensen, C.S., Xu, G. (eds.) APWeb 2012, LNCS, vol. 7235, pp. 318-329. Springer, Heidelberg (2012)

22. Juels, A., Weis, S.A.: Defining strong privacy for RFID. ACM Trans. Inf. Syst. Secur. 13(1) (2009)

23. Jung, S.W., Jung, S.: HRP: A HMAC-based RFID mutual authentication protocol using PUF. In: ICOIN 2013. pp. 578-582. IEEE (2013)

24. Krishna, A.R., Narasimhan, S., Wang, X., Bhunia, S.: MECCA: A robust lowoverhead PUF using embedded memory array. In: Preneel, B., Takagi, T. (eds.) CHES 2011. LNCS, vol. 6917, pp. 407-420. Springer, Heidelberg (2011)

25. Kulseng, L., Yu, Z., Wei, Y., Guan, Y.: Lightweight mutual authentication and ownership transfer for RFID systems. In: INFOCOM, 2010 Proceedings IEEE. pp. 251-255. IEEE (2010)

26. Lee, M.Z., Dunn, A.M., Katz, J., Waters, B., Witchel, E.: Anon-pass: Practical anonymous subscriptions. IEEE Security \& Privacy 12(3), 20-27 (2014)

27. Maes, R.: Physically Unclonable Functions - Constructions, Properties and Applications. Springer (2013)

28. Maes, R., van der Leest, V.: Countering the effects of silicon aging on SRAM PUFs. In: HOST 2014. pp. 148-153. IEEE (2014)

29. Majzoobi, M., Rostami, M., Koushanfar, F., Wallach, D.S., Devadas, S.: Slender PUF protocol: A lightweight, robust, and secure authentication by substring matching. In: IEEE Security \& Privacy. pp. 33-44. IEEE (2012)

30. Moriyama, D., Matsuo, S., Yung, M.: PUF-based RFID authentication secure and private under complete memory leakage. IACR Cryptology ePrint Archive 2013, 712 (2013), http://eprint.iacr.org/2013/712

31. Nedospasov, D., Seifert, J., Helfmeier, C., Boit, C.: Invasive PUF analysis. In: Fischer, W., Schmidt, J. (eds.) FDTC 2013. pp. 30-38. IEEE (2013)

32. Oren, Y., Sadeghi, A., Wachsmann, C.: On the effectiveness of the remanence decay side-channel to clone memory-based PUFs. In: Bertoni, G., Coron, J. (eds.) CHES 2013. LNCS, vol. 8086, pp. 107-125. Springer, Heidelberg (2013)

33. Paral, Z.S., Devadas, S.: Reliable and efficient PUF-based key generation using pattern matching. In: HOST 2011. pp. 128-133. IEEE (2011)

34. Rührmair, U., Sölter, J., Sehnke, F., Xu, X., Mahmoud, A., Stoyanova, V., Dror, G., Schmidhuber, J., Burleson, W., Devadas, S.: PUF modeling attacks on simulated and silicon data. IEEE Transactions on Information Forensics and Security 8(11), 1876-1891 (2013)

35. Rührmair, U., Xu, X., Sölter, J., Mahmoud, A., Majzoobi, M., Koushanfar, F., Burleson, W.P.: Efficient power and timing side channels for physical unclonable functions. In: Batina, L., Robshaw, M. (eds.) CHES 2014. LNCS, vol. 8731, pp. 476-492. Springer, Heidelberg (2014)

36. Rukhin, A., Soto, J., Nechvatal, J., Smid, M., Barker, E., Leigh, S., Levenson, M., Vangel, M., Banks, D., Heckert, A., Dray, J., Vo, S.: A statistical test suite for the validation of random number generators and pseudo random number generators for cryptographic applications. Special Publication 800-22 Revision 1a (April 2010)

37. Sadeghi, A.R., Visconti, I., Wachsmann, C.: Enhancing RFID security and privacy by physically unclonable functions. In: Towards Hardware-Intrinsic Security, pp. 281-305. Springer, Heidelberg (2010) 
38. Van Herrewege, A., Katzenbeisser, S., Maes, R., Peeters, R., Sadeghi, A., Verbauwhede, I., Wachsmann, C.: Reverse fuzzy extractors: Enabling lightweight mutual authentication for PUF-enabled RFIDs. In: Keromytis, A.D. (ed.) FC 2012. LNCS, vol. 7397, pp. 374-389. Springer, Heidelberg (2012)

39. Yu, M.D., M'Raïhi, D., Devadas, S., Verbauwhede, I.: Security and reliability properties of syndrome coding techniques used in PUF key generation. In: 38th GOMACTech conference. pp. 1-4 (2013)

\section{A Security Analysis}

\section{A.1 Security Model for Anonymous Authentication Protocols}

We follow the security model in [30] and describe the theoretical security and privacy.

Communication Model. Consider that there is one trustworthy server $\mathcal{S}$ and $n$ devices. We denote a set of device by $\operatorname{Dev}:=\left\{\operatorname{Dev}_{0}, \ldots, \operatorname{Dev}_{n}\right\}$. For initialization, the server runs a setup algorithm $\operatorname{Setup}\left(1^{k}\right)$ and generates public parameter $p p$ and secret keys $s k$. In some cases, the server communicates with devices in a secure environment and transfers the critical information to start the authentication. When the authentication phase is started, these parties interact in an insecure network and authenticate each other. Finally, the parties output 1 (acceptance) or 0 (rejection) as the authentication result, respectively. A communication sequence between them is called a session, and each session is distinguished by session identifier sid which contains a series of communication message. We say that a session has a matching session if the communication messages generated by the server and the device are honestly transferred until they authenticate each other.

Security. The well-known highest security level for authentication protocols is the resilience to the man-in-the-middle attack. The authentiation results for both parties becomes 1 if and only if the communication message is honestly transferred. In addition to the canonical security requirement for the man-inthe-middle attack, we allow the adversary can obtain the memory content in the non-volatile memory before and after the session (authentication) in our model.

In a theoretical level, the security is evaluated by the following experiment between a challenger and adversary $\mathcal{A}$.

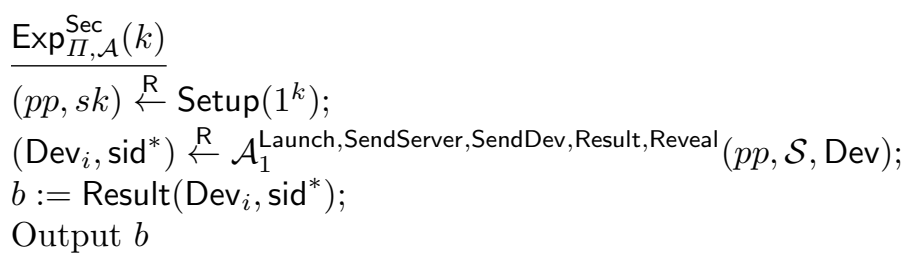

After the setup phase, the adversary $\mathcal{A}$ can interact with the server and device and obtain several information with the following oracle queries: 
- Launch $\left(1^{k}\right)$ : Launch the server to start a new session.

- SendServer $(m)$ : Send arbitrary message $m$ to the server.

- SendDev $\left(\operatorname{Dev}_{i}, m\right)$ : Send arbitrary message $m$ to the device $\operatorname{Dev}_{i} \in \operatorname{Dev}_{\text {. }}$

- $\operatorname{Result}(P$, sid): Output whether the session sid of $P$ is accepted or not where $P \in\{\mathcal{S}, \operatorname{Dev}\}$.

- Reveal $\left(\operatorname{Dev}_{i}\right)$ : Output whole information contained in the memory in $\operatorname{Dev}_{i}$.

The advantage of adversary $\mathcal{A}$ against $\Pi, \operatorname{Adv}_{\Pi, \mathcal{A}}^{\mathrm{Sec}}(k)$, is defined by probability that $\operatorname{Exp}_{I I, \mathcal{A}}^{\mathrm{Sec}}(k)$ outputs 1 on the condition that sid* of $P$ has no matching session.

Definition 1. An authentication protocol $\Pi$ holds the security against man-inthe-middle attack with key compromise if for any probabilistic polynomial time adversary $\mathcal{A}, \operatorname{Adv}_{\Pi, \mathcal{A}}^{\mathrm{Sec}}(k)$ is negligible in $k$ (for large enough $k$ ).

Privacy. The privacy definition described in [30] is based on the indistinguishabilitybased privacy such that the adversary selects two devices and tries to distinguish the communication derived from one of the two devices. The privacy experiment between a challenger and adversary $\mathcal{A}:=\left(\mathcal{A}_{1}, \mathcal{A}_{2}, \mathcal{A}_{3}\right)$ is the following:

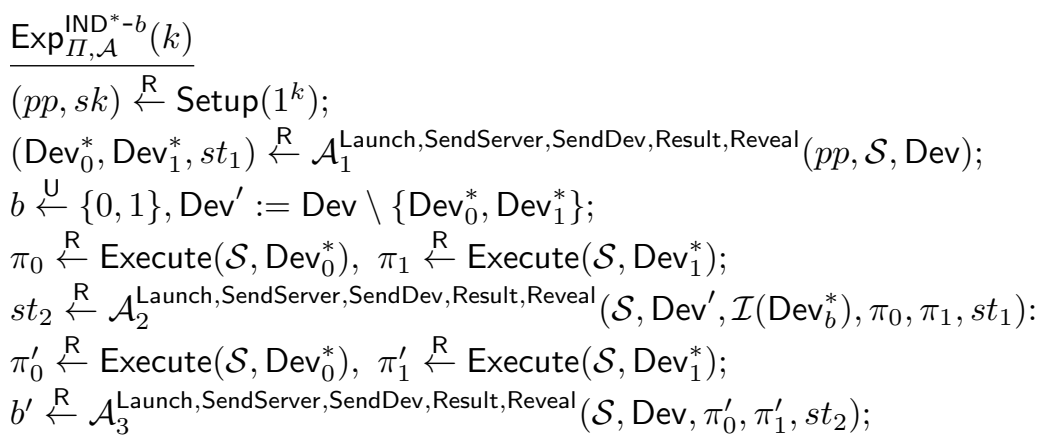

After the setup phase, the adversary issues the oracle queries as the security experiment and submits $\left(\operatorname{Dev}_{0}^{*}, \operatorname{Dev}_{1}^{*}\right)$ to the challenger. The challenger flips a bit $b \stackrel{U}{\leftarrow}\{0,1\}$ and allows the adversary to interact with $\operatorname{Dev}_{b}^{*}$ in an anonymous way. This anonymous interaction is accomplished by adding a special identity $\mathcal{I}$ who honestly transfers the communication message between $\mathcal{A}$ and $\operatorname{Dev}_{b}^{*}$. To avoid a trivial attack in the symmetric key based construction, both $\operatorname{Dev}_{0}^{*}$ and $\operatorname{Dev}_{1}^{*}$ execute a honest session with the server denoted by Execute before and after the anonymous interaction and its transcripts are sent to the adversary. The advantage of the adversary is defined as

$$
\operatorname{Adv}_{\Pi, \mathcal{A}}^{\mathrm{IND}}(k):=\left|\operatorname{Pr}\left[\operatorname{Exp}_{\Pi,, \mathcal{A}}^{\mathrm{INN} D^{*}-0}(k) \rightarrow 1\right]-\operatorname{Pr}\left[\operatorname{Exp}_{\Pi,, \mathcal{A}}^{\mathrm{INN} D^{*}-1}(k) \rightarrow 1\right]\right| .
$$

Definition 2. An authentication protocol $\Pi$ holds forward and backward privacy if for any probabilistic polynomial time $(P P T)$ adversary $\mathcal{A}, \operatorname{Adv}_{I I, \mathcal{A}}^{\mathbf{I N D}}(k)$ is negligible in $k$ (for large enough $k$ ). 


\section{A.2 Formal Security Definition for Building Blocks}

Toward the provable security, we show the detailed security definition for each building blocks used in our protocols.

Physically Unclonable Functions (PUFs). $f: \mathcal{K} \times \mathcal{D} \rightarrow \mathcal{R}$ is a physically unclonable function which takes as input a physical chatacterics implemented in the device $x \in \mathcal{K}$ and input message $y \in \mathcal{D}$ and outputs $z \stackrel{R}{\leftarrow} f(x, y)$. To dinstinguish multiple devices, we denote PUF as $f\left(x_{1}, \cdot\right), f\left(x_{2}, \cdot\right), \ldots$. We say that the PUF is $(n, \ell, d, h, \epsilon)$-secure PUF if the following conditions hold:

1. For any PUF $f\left(x_{1}, \cdot\right)$ and for any input $y_{1} \in \mathcal{D}$,

$$
\operatorname{Pr}\left[\operatorname{HD}\left(f\left(x_{1}, y_{1}\right), f\left(x_{1}, y_{1}\right)\right)<d\right]=1-\epsilon .
$$

2. For any PUFs $f\left(x_{1}, \cdot\right), f\left(x_{2}, \cdot\right)$ and for any input $y_{1} \in \mathcal{D}$,

$$
\operatorname{Pr}\left[\operatorname{HD}\left(f\left(x_{1}, y_{1}\right), f\left(x_{2}, y_{1}\right)\right)>d\right]=1-\epsilon .
$$

3. For any PUF $f\left(x_{1}, \cdot\right)$ and for any inputs $y_{1}, y_{2} \in \mathcal{D}$,

$$
\operatorname{Pr}\left[\operatorname{HD}\left(f\left(x_{1}, y_{1}\right), f\left(x_{1}, y_{2}\right)\right)>d\right]=1-\epsilon .
$$

4. For any PUFs $f\left(x_{1}, \cdot\right), \ldots, f_{\left.x_{n}, \cdot\right)}$ and for any inputs $y_{1}, \ldots, t_{\ell} \in \mathcal{D}$,

$$
\operatorname{Pr}\left[\tilde{H}_{\infty}\left(f\left(x_{i^{*}}, y_{j^{*}}\right)\left\{f\left(x_{i}, y_{j}\right)\right\}_{1 \leq n, 1 \leq j \leq \ell, i \neq i^{*}, j \neq j^{*}}>h\right]=1-\epsilon .\right.
$$

These formulations provide that the intra-distance is smaller than $d$, inter distance (from two metrics) is larger than $d$ and (conditional) min-entropy of the PUF is always larger than $h$.

Definition 3. A PUF satisfies $(n, \ell, d, h, \epsilon)$-secure PUF if all the above conditions hold.

Fuzzy Extractor. A $(d, h, \epsilon)$-fuzzy extractor FE consists of two algorithms: key generation algorithm FE.Gen and reconstruction algorithm FE.Rec. The FE.Gen algorithm takes as input a variable $z$ and output a key $r$ and helper data $h d$. For correctness, FE. Rec recovers the key $r$ from the input variable $z$ and helper data $h d$ if the hamming distance between $z$ and $z^{\prime}$ is at most $d$. The fuzzy extractor satisfies security if the min-entropy of input $z$ is at least $h, r$ is statistically $\epsilon$-close to a uniformly random variable in $\{0,1\}^{k}$, even if the helper data is disclosed.

Definition 4. A fuzzzy extractor satisfies $(d, h, \epsilon)$-fuzzy extractor if the following conditions hold:

1. $\operatorname{Pr}\left[r:=\mathrm{FE} \cdot \operatorname{Rec}\left(z^{\prime}, h d\right) \mid(r, h d) \stackrel{\mathrm{R}}{\leftarrow} \mathrm{FE} \cdot \operatorname{Gen}(z), \operatorname{HD}\left(z, z^{\prime}\right) \leq d\right]=1$.

2. If $\tilde{H}_{\infty}(z) \geq h,(r, h d) \stackrel{R}{\leftarrow} \mathrm{FE}$.Gen $(z)$ is statistically $\epsilon$-close from $\left(r^{\prime}, h d\right)$ where $r^{\prime} \stackrel{\cup}{\leftarrow}\{0,1\}^{|r|}$. 
Pseudorandom Function (PRF). A pseudorandom function PRF : $\{0,1\}^{k} \times$ $\{0,1\}^{*} \rightarrow\{0,1\}^{k^{\prime}}$ which takes as input a secret key $s k \in\{0,1\}^{k}$ and input $m \in\{0,1\}^{*}$ and provides an arbitrary string $\operatorname{PRF}(s k, m)$ which is indistinguishable from random string. The security of the PRF is defined by the following experiment between a challenger and adversary $\mathcal{A}$. First, the challenger selects coin $b \stackrel{U}{\leftarrow}\{0,1\}$ and secret key $s k \stackrel{\cup}{\leftarrow}\{0,1\}^{k}$. The challenger also prepares a truly random function RF. The adversary $\mathcal{A}$ can adaptively issue a oracle query to the challenger to obtain a response of a function. When $\mathcal{A}$ sends $m$, the challenger responds $\operatorname{PRF}(s k, m)$ if $b=1$. On the other hand, if $b=0$, the challenger inputs $m$ to RF and responds its result. Finally, the adversary outputs a guess $b^{\prime}$. If $b^{\prime}=b$, the adversary wins the experiment. The advantage of the adversary to win the experiment is defined by $\operatorname{Adv}_{\mathcal{A}}^{\mathrm{PRF}}(k)=\left|2 \cdot \operatorname{Pr}\left[b^{\prime}=b\right]-1\right|$.

Definition 5. A PRF is $\epsilon$-secure PRF for any probabilistic polynomial time adversary $\mathcal{A}, \operatorname{Adv}_{\mathcal{A}}^{\mathrm{PRF}}(k) \leq \epsilon$ holds.

\section{A.3 Security Proof for the Proposed Protocol}

Theorem 1. Let PUF $f$ be a $\left(n, \ell, d, h, \epsilon_{1}\right)$-secure PUF, FE be a $\left(d, h, \epsilon_{2}\right)$-fuzzy extractor, PRF be a $\epsilon_{3}$-secure PRF. Then our protocol $\Pi$ holds security against man-in-the-middle attack with key compromise. Especially, we have $\operatorname{Adv}_{I, \mathcal{A}}^{\mathrm{Sec}} \leq$ $\ln \left(\epsilon_{1}+\epsilon_{2}+\epsilon_{3}\right)$.

Proof. The goal of the adversary $\mathcal{A}$ is to violate the security experiment and it means that the server or the device accepts the session without maching session. Let $S_{i}$ be the advantage that the adversary wins the game in Game $i$. We consider the following game transformations.

Game 0. This is the original game between the challenger and the adversary.

Game 1. The challenger randomly guesses the device $\operatorname{Dev}^{*} \stackrel{\cup}{\leftarrow}$ Dev. If $\operatorname{Dev}^{*}$ does not participate in sid*, the challenger aborts the game.

Game 2. Assume that $\ell$ is the upper bound of the sessions that the adversary can establish in the game. For $1 \leq j \leq \ell$, we transform the variables related to the the session between the server and $\operatorname{Dev}^{*}$ up to the $\ell$-th session as the following.

Game 2-j-1. The challenger evaluates the output from the PUF implemented in $\mathrm{Dev}^{*}$ at the $j$-th session. If the intra-distance is larger than $d$, inter-distance is smaller than $d$ or min-entropy of the output is smaller than $d$, the challenger aborts the game.

Game 2-j-2. The output from the fuzzy extractor $r_{1}$ is changed to a random variable.

Game 2-j-3. The output from the $\operatorname{PRF} \operatorname{PRF}\left(r_{1}, \cdot\right)$ is derived from a truly random function.

Game 2-j-4. The output from the $\operatorname{PRF} \operatorname{PRF}\left(r_{\text {old }}, \cdot\right)$ is derived from a truly random function.

Game 2-j-5. We change the XORed output $u_{1}:=t_{2} \oplus z_{2}^{\prime}$ to randomly chosen $u_{1} \stackrel{\cup}{\leftarrow}\{0,1\}^{\left|u_{1}\right|}$. 
Game 2- $j$-6. The output from the $\operatorname{PRF}_{\operatorname{PRF}}\left(t_{3}, \cdot\right)$ is derived from a truly random function.

Lemma 1. $S_{0}=n \cdot S_{1}$ (where $n$ is the number of devices).

The violation of security means that there is a session which the server or device accepts the session while the communication is modified by the adversary. Since we assume that the number of device is at most $n$, the challenger can correctly guess the related session with probability at least $1 / n$.

Lemma 2. $\left|S_{1}-S_{2-1-1}\right| \leq \epsilon_{1}$ and $\left|S_{2-(j-1)-6}-S_{2-j-1}\right| \leq \epsilon_{1}$ for any $2 \leq j \leq \ell$ if the PUF is $\left(n, \ell, d, h, \epsilon_{1}\right)$-secure PUF.

We now assume that the PUF satisfies $\left(n, \ell, d, h, \epsilon_{1}\right)$-secure PUF in advance. This means the intra-distance is less than $d$, inter-distance is larger than $d$ and min-entropy of the PUF is always larger than $h$ except the negligible probability $\epsilon$. Since $S_{1}$ and $S_{2-(j-1)-6}$ assume the condition except the negligible probability $\epsilon$ and $S_{2-1-1}$ and $S_{2-j-1}$ requires the condition with probability 1, respectively, the gap between them is bounded by $\epsilon_{1}$.

Lemma 3. $\forall 1 \leq j \leq \ell,\left|S_{2-j-1}-S_{2-j-2}\right| \leq \epsilon_{2}$ if the FE is a $\left(d, h, \epsilon_{2}\right)$-fuzzy extractor.

From the proof of Lemma 2, we can assume that the PUF provides enough minentropy $h$. Then the property of the $(d, h)$-fuzzy extractor guarantees that the output for the fuzzy extractor is statistically close to random and no adversary can distinguish the difference between the two games.

Lemma 4. $\forall 1 \leq j \leq \ell,\left|S_{2-j-2}-S_{2-j-3}\right| \leq \operatorname{Adv}_{\mathcal{B}}^{\mathrm{PRF}}(k)$ for a PPT algorithm $\mathcal{B}$.

Lemma 5. $\forall 1 \leq j \leq \ell,\left|S_{2-j-3}-S_{2-j-4}\right| \leq \operatorname{Adv}_{\mathcal{B}}^{\mathrm{PRF}}(k)$ for a PPT algorithm $\mathcal{B}$.

If there is a difference between these games, we construct the algorithm $\mathcal{B}$ which breaks the security or $\operatorname{PRF} \mathcal{G} \cdot \mathcal{B}$ can access the real $\operatorname{PRF} \operatorname{PRF}\left(r_{1}, \cdot\right)$ or truly random function RF. $\mathcal{B}$ sets up all secret keys and simulates our protocol except the $n$-th session. When the adversary invokes the $n$-th session, $\mathcal{B}$ sends $y_{1} \stackrel{U}{\leftarrow}$ $\{0,1\}^{k}$ as the output of the server. When $\mathcal{A}$ sends $y_{1}^{*}$ to a device $\operatorname{Dev}_{i}, \mathcal{B}$ selects $y_{2}^{\prime}$ and issues $y_{1}^{*} \| y_{2}^{\prime}$ to the oracle instead of the normal computation of $\mathcal{G}$. Upon receiving $\left(t_{1}, \ldots, t_{5}\right), \mathcal{B}$ continues the computation as the protocol specification and outputs $\left(c, y_{2}^{\prime}, t_{1}, u_{1}, v_{1}\right)$ as the device's response. When the adversary sends $\left(y_{2}^{*}, t_{1}^{*}, u_{1}^{*}, v_{1}^{*}\right), \mathcal{B}$ issues $y_{1} \| y_{2}^{*}$ to the oracle and obtains $\left(t_{1}^{\prime}, \ldots, t_{5}^{\prime}\right)$.

If $\mathcal{B}$ accesses the real PRF, this simulation is equivalent to Game $2-j-2$. Otherwise, the oracle query issued by $\mathcal{B}$ is completely random and this distribution is equivalent to Game $2-j-3$. Thus we have $\left|S_{2-j-2}-S_{2-j-3}\right| \leq \operatorname{Adv}_{\mathcal{B}}^{\mathrm{PRF}}(k)$.

Lemma 6. $\forall 1 \leq j \leq \ell, S_{2-j-4}=S_{2-j-5}$.

Since $t_{2}$ is already changed to the random string, no adversary can find the difference from $u_{1}:=t_{1} \oplus z_{2}^{\prime}$ from randomly chosen string. 
Lemma 7. $\forall 1 \leq j \leq \ell,\left|S_{2-j-5}-S_{2-j-6}\right| \leq 2 \cdot \operatorname{Adv}_{\mathcal{B}}{ }^{\mathrm{RF}^{\prime}}(k)$ for a PPT algorithm $\mathcal{B}$.

Consider an algorithm $\mathcal{B}$ which interacts with $\operatorname{PRF}_{\operatorname{PRF}^{\prime}}\left(t_{3}, \cdot\right)$ or random function RF. $\mathcal{B}$ runs setup algorithm and simulates the protocol up to the $n$-th session. After computing $\left(c, u_{1}\right)$ and issues $c \| u_{1}$ to the oracle. $\mathcal{B}$ generates the other variables as the previous game and sends $\left(c, y_{2}^{\prime}, t_{1}, u_{1}, v_{1}\right)$ as the device's output after it obtains $v_{1}$ from the oracle. If the server receives $\left(c^{*}, y_{2}^{*}, t_{1}^{*}, u_{1}^{*}, v_{1}^{*}\right), \mathcal{B}$ checks that $\left(c^{*}, y_{2}^{*}\right)=\left(c, y_{2}^{\prime}\right)$. If so, $\mathcal{B}$ issues $c^{*} \| y_{1}^{*}$ to the oracle to check whether its response is identical to $v_{1}^{*}$.

If $\mathcal{B}$ accesses the real PRF, this simulation is equivalent to Game $2-j-5$. Otherwise, $\mathcal{B}$ 's simulation is identical to Game $2-j-6$. Thus the difference between these games are bounded by the security of PRF $\mathcal{G}^{\prime}$. Similarly, we can evaluate the gap between $\operatorname{PRF}^{\prime}\left(t_{3}^{\prime}, \cdot\right)$ and RF.

Since the above game transformation is bounded by certain assumptions (for PUF, fuzzy extractor and PRFs), we can transform Game 0 to Game 2- $\ell$ 6 . When we consider Game $2-\ell-6$, it is impossible for the adversary to violate the security. Consider the case that the server accepts the session which is not actually derived the device. Assume that the adversary obtains $\left(c, y_{2}^{\prime}, t_{1}, u_{1}, v_{1}\right)$ from the device. To mount the man-in-the-middle attack, the adversary must modify at least one of these variables. Even when the adversary issues the reveal query and obtains $\left(s k, y_{1}\right)$ before the session, it only reveals $h d_{1}$ decrypted by $c$ and it is hard for the adversary to estimate $r_{1}$ from the property of the fuzzy extractor. While the adversary can select arbitrary message $h d^{\prime}$ and compute a ciphertext $c^{\prime}:=\operatorname{SKE}$.Enc $\left(s k, h d^{\prime}\right)$ which is actually decrypted by the server, it is hard for the adversary to generate valid $v_{1}^{\prime}=\operatorname{PRF}^{\prime}\left(t_{3}, c^{\prime} \| u_{1}\right)$ without $t_{3}$ (due to the pseudorandomness from PRF). Similarly, if the adversary changes $y_{2}^{\prime}$ or $u_{1}$, the pseudorandom function is already changed to the truly random function and it is infeasible for the adversary to generate a valid $\left(t_{1}, v_{1}\right)$ directly corresponding to these variables. Therefore, no adversary cannot mount the man-in-the-middle attack in our protocol.

Theorem 2. Let PUF $f$ be $a\left(n, \ell, d, h, \epsilon_{1}\right)$-secure PUF, FE be $a\left(d, h, \epsilon_{2}\right)$-fuzzy extractor and PRF be a $\epsilon_{3}$-secure PRF. Then our protocol holds forward and backward privacy.

Proof. The proof we provide here is similar to that for Theorem 1. We first assume that our protocol holds security. Otherwise, the adversary desynchronizes the secret key between the server and gadget over the resynchronization capability. Then the server continuously reject message came from the gadgets and the adverary easily distinguishes the server interacts with the ligitimate or desynchronized device. Since we already proved Theorem ??, this concern is cleared.

The remained proof strategy is quite similar to the proof of Theorem 1 . We continuously change the communication message for the target devices $\operatorname{Dev}_{0}^{*}$ $\operatorname{Dev}_{1}^{*}$. Then the whole message transcripts looks like random strings and no 
biased information which identifies the challenger's coin is leaked. Again, memory content $\left(s k, y_{1}\right)$ does not provide any information about the target device since it is generated by PRF or selected from random. The challenger can estimate $\left(\operatorname{Dev}_{0}^{*}, \mathrm{Dev}_{1}^{*}\right)$ with probability at least $1 / n^{2}$ so the game transformation is finished within a polynomial time. Therefore. we can say that the proposed protocol satisfies the forward and backward privacy. 